\title{
Acute Ophthalmoplegia; Same Disease, Different Variants: Anti GQ1b Antibody Syndrome
}

\author{
(1) Senem Ayça1, (1) Anna Carina Ergani2, (1) Muzaffer Polat1 \\ ${ }^{1}$ Manisa Celal Bayar University Faculty of Medicine, Department of Pediatric Neurology, Manisa, Turkey \\ 2 Manisa Celal Bayar University Faculty of Medicine, Department of Pediatrics, Manisa, Turkey
}

\begin{abstract}
Patients with Miller Fisher syndrome (MFS) are characterized by acute ophthalmoplegia (AO) and areflexia. MFS is an immune mediated process, triggered by an infection and includes incomplete forms, such as ophthalmoplegia, ataxia and a central nervous system subtype known as Bickerstaff brainstem encephalitis (BBE). We present two cases admitted to our hospital on the same day. The first case was presented as AO, with elevated levels of anti GQ1b. The second case was presented as AO, oropharyngeal palsy and sensory motor polyneuropathy with borderline levels of anti GQ1b, diagnosed as BBE. There are atypical forms of MFS with different clinical symptoms and elevated levels of antibodies called "Anti CQ1b Antibody syndrome". The cases of the two patients diagnosed as MFS variants' AO and BBE.
\end{abstract}

Keywords: Bickerstaff brainstem encephalitis, Miller Fisher syndrome, acute ophthalmoplegia, anti GQ1b antibody

\section{Introduction}

Guillain-Barrē syndrome (CBS) is characterized by muscle weakness and a loss of deep tendon reflexes (1). Miller Fisher syndrome (MFS) is a variant of GBS and its clinical triad includes ophthalmoplegia, ataxia and areflexia. It is likely that CBS, MFS and Bickerstaff brainstem encephalitis (BBE) form a continuous spectrum. MFS includes incomplete forms, such as acute mydriasis, acute ataxic neuropathy, acute oropharyngeal palsy, AO and BBE (2). In this report we present two cases admitted to hospital on the same day presenting acute ophthalmoplegia diagnosed as BBE and AO.

\section{Case Reports}

\section{Case 1}

A 3-year old previously healthy girl was admitted to hospital due to difficulty in walking and sitting, hoarseness and a deviation of bilateral eyes. Two days prior to admittance, she experienced sudden bilateral eye deviation and subsequently her clinical status worsened so that she could not walk or sit without support. Three weeks before the admission, she had been diagnosed with acute tonsillitis. On admission, somnolence and sudden hoarseness developed and signs of meningeal irritation were positive. Deep tendon reflexes were active and the Babinski sign was positive bilaterally. She had bilateral sixth nerve palsy (Figure 1). Her muscle strength was diminished in all extremities (medical research council: $3 / 5$ ). The rest of her general physical examination was unremarkable. Brain and spine magnetic resonance (MR) imaging detected no abnormal signals. Cerebrospinal fluid (CSF) cytochemical analysis findings were as follows; no cells detected, protein $104 \mathrm{mg} / \mathrm{dL}$, glucose $56 \mathrm{mg} / \mathrm{dL}$ confirming albumin cytological dissociation. Serological CSF tests were negative for Mycoplasma pneumoniae, 

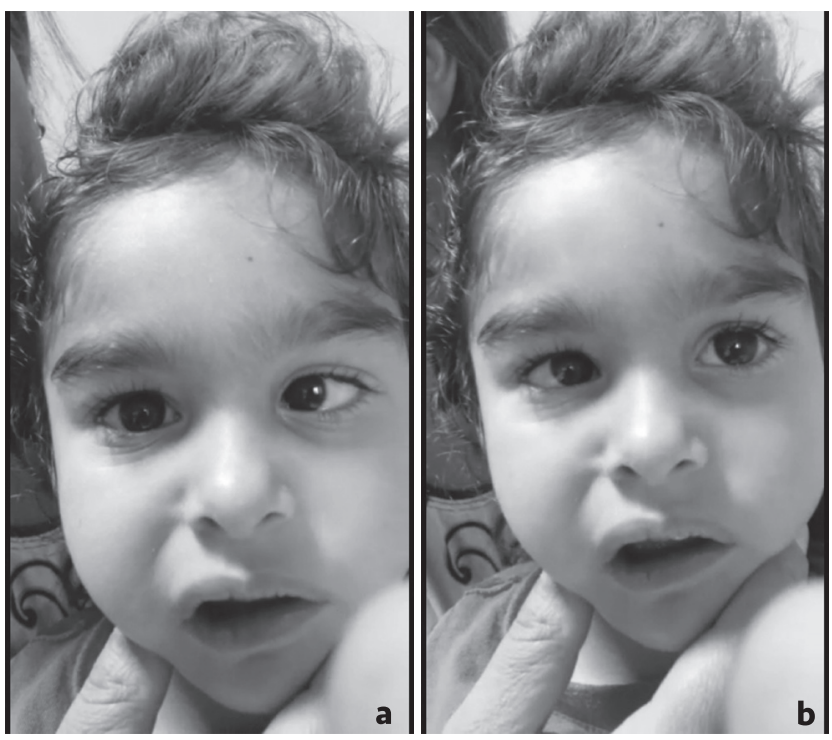

Figure 1. a) Left eye abduction paresis, b) right eye abduction paresis

Enterovirus, Cytomegalovirus and Epstein Barr virus. Blood and CSF cultures were sterile. Five days after admission, electromyoneurography revealed sensory motor axonal polyneuropathy and electrophysiological studies revealed an absence of $\mathrm{H}$ reflex. Intravenous immunoglobulin (IVIC) therapy $(2 \mathrm{gr} / \mathrm{kg}$ ) was administrated one day after admission. The symptoms of hoarseness and somnolence regressed but she was unable to do coordination tests adequately and her gait was abnormal. She had truncal ataxia and could not sit or walk without support. Her meningeal irritation symptoms were still positive. Pulse steroid therapy was started, two days after the therapy she clinically and could sit and walk without any support. Her meningeal irritation signs were then seen to be negative. Corticosteroid treatment (2 mg/ $\mathrm{kg} / \mathrm{d}$ ) was maintained for 4 weeks. One month after her discharge, her neurologic examination was normal. Testing for anti-ganglioside antibodies resulted in borderline levels of serum anti GQ1b antibodies. Our patient was diagnosed with $\mathrm{BBE}$, including impaired consciousness, muscle weakness with positive CSF and electrophysiological findings. Informed consent was obtained from the patient's parents.

\section{Case 2}

A 6-year-old previously healthy girl was admitted to hospital with a four-day history of diplopia. A few weeks previously, the child had an episode consisting of coughing, fever, diarrhea and vomiting. On admission, she was afebrile and well oriented. Her light reflex was normal. She had limited abduction, adduction, up-gaze and downgaze paresis of the right eye and abduction paresis of the left eye (Figure 2). Her deep tendon reflexes and muscle strength were normal. The Babinski sign was negative. The results of blood investigation were normal. A lumbar puncture was performed, CSF cytochemical analysis showed

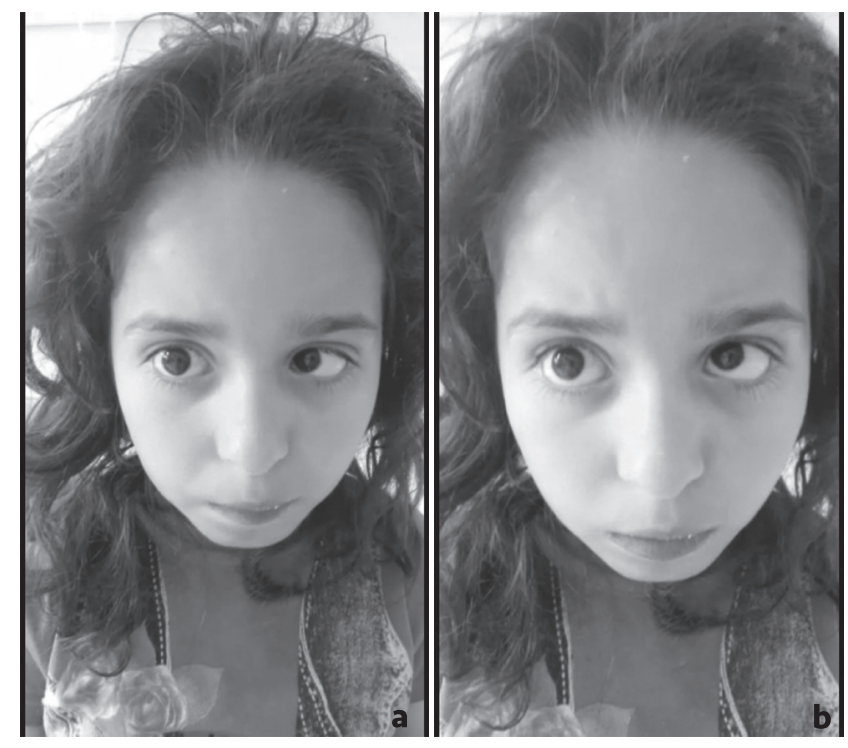

Figure 2. a) Abduction, adduction, up-gaze and down-gaze paresis of the right eye, b) abduction paresis of the left eye

no abnormality. Serological CSF tests were negative for Mycoplasma pneumoniae, Enterovirus, Cytomegalovirus and Epstein Barr virus. Blood and CSF cultures were sterile. Electrophysiological studies were normal. Anti-acetylcholine receptor antibodies were negative. Cerebral imaging by MR and MR angiography revealed no abnormality. Testing for anti-ganglioside antibody panel were done and revealed high levels of serum anti GQ1b++ and GD1b+. IVIG therapy was started six days after the onset of the symptoms. Two days after starting IVIG therapy, paresis of up-down gaze and adduction of the right eye regressed and movements of the left eye were normal. Diplopia proceeded and steroid therapy ( $2 \mathrm{mg} / \mathrm{kg} /$ day) was administered for four weeks. One month after her discharge, her neurologic examination was almost normal except for limited abduction paresis of the right eye.

Informed consent was obtained from the patient's parents.

\section{Discussion}

MFS is an inflammatory neuropathy with clear consciousness and BBE is a central nervous system disease characterized by consciousness disturbance. Acute ophthalmoplegia can be seen in both BBE and MFS and it is associated with the presence of ganglioside GQ1b and called Anti GQ1b Antibody syndrome $(3,4)$. We describe two patients admitted at the same time with bilateral ophthalmoplegia. One of the patients had isolated $A O$ with high levels of anti GQ1b. The other case had additional symptoms; impaired consciousness, $\mathrm{AO}$, hyperreflexia, neck stiffness, pharyngeal palsy and positive electrophysiological findings (sensorimotor polyneuritis, radiculopathy, absence of $\mathrm{H}$ reflex). Because of impaired consciousness and 
sensorimotor polyneuritis meant both peripheral and central nervous system involvement. She had borderline levels of anti GQ1b. We speculated that taking a blood sample after the initiation of steroid treatment could be responsible for the low levels of anti GQ1b. Akinci et al. (5) described an MFS case with a pattern of pure sensory polyneuropathy concomitant with anti GQ1B antibody. For patients with acute isolated ophthalmoplegia with an unknown etiology, Anti GQ1b Antibody syndrome should be considered and anti-ganglioside antibody panel testing should be done. Also testing for this panel could be done in case of an unknown etiology of encephalitis; diagnosis of BBE can be helpful in the administration of the appropriate treatment.

Ethics

Informed Consent: Consent form was filled out by all participants.

Peer-review: External and internal peer-reviewed.

\section{Authorship Contributions}

Surgical and Medical Practices: M.P., S.A., Concept: M.P., Design: M.P., Data Collection or Processing: S.A., A.C.E., Analysis or Interpretation: M.P., S.A., Literature Search: A.C.E., S.A., Writing: S.A., A.C.E.
Conflict of Interest: No conflict of interest was declared by the authors.

Financial Disclosure: The authors declared that this study received no financial support.

\section{References}

1. Fisher M. An unusual variant of acute idiopathic polyneuritis syndrome of ophtalmoplegia, ataxia and areflexia. N Engl / Med 1956;255:57-65.

2. Aranyi Z, Kovacs T, Sipos I, Bereczki D. Miller fisher syndrome: Brief overview and update with a focus on electrophysiological findings. Eur / Neurol 2012;19:15-20.

3. Guisset F, Ferreiro C, Voets S, et al. Anti-GQ1b antibody syndrome presenting as acute isolated bilateral ophthalmoplegia: Report on two patients and review of the literature. Eur I Paediatr Neurol 2016;20:439-43.

4. Yuki N, Sato S, Tsuji S, Hozumi I, Miyatake T. An immunologic abnormality common to Bickerstaff's brainstem encephalitis and Fisher's syndrome. J Neurol Sci 1993;118:83-7.

5. Akinci G, Polat M, Tosun A, Serdaroğlu G, Gökben S, Tekgül H. Miller Fisher syndrome: a case with pattern of pure sensory polyneuropathy concomitant with anti-GQ1B antibody. Turk I Pediatr 2007;49:109-12. 